\title{
On Variational Principles for Coherent Vortex Structures
}

\author{
B.W. VAN DE FLIERT and E. VAN GROESEN \\ Dept. of Appl. Math., University of Twente, The Netherlands
}

\begin{abstract}
Different approaches are discussed of variational principles characterizing coherent vortex structures in two-dimensional flows. Turbulent flows seem to form ordered structures in the large scales of the motion and the self-organization principle predicts asymptotic states realizing an extremal value of the energy or a minimum of enstrophy. On the other hand the small scales take care of the increase of entropy, and asymptotic results can be obtained by applying the theory of equilibrium statistical mechanics.
\end{abstract}

Key words: plane vorticity flows - variational methods - relative equilibria - self-organization

\section{Introduction}

Numerical and laboratory experiments reveal that in two-dimensional turbulent flows most of the vorticity gets concentrated inside long-living vortices, which seem to represent the relevant part of the dynamics of the system. The behaviour of the flow is dominated by large-scale motion, when isolated eddies have formed due to merging of like-sign vortices and dipole formation of opposite-sign vortices. To describe the asymptotic behaviour of the turbulent flow it seems important to understand the dynamics and stability of single and several interacting isolated structures.

If due to a high Reynolds number the dynamics is dominated by the inertial terms in the evolution equation, one can make the assumption that the system is described by the inviscid Euler equations. Indeed, special critical solutions of the conservative system correspond very well to the observed coherent states and one might expect that a small dissipation will only distort the dynamics slightly. The inviscid Euler equations and the critical solutions are subject of the first part of this review. In the second part we will discuss the adiabatic decay of the coherent structures. Applying the so-called self-organization hypothesis, the relative equilibria have been shown to describe the asymptotic behaviour of the dissipative system $[7,5]$. In contrast with the deterministic self-organization towards relative equilibria, studies have been made on the emergence of isolated vortex structures by considering statistical equilibrium solutions. In this review we want to compare different approaches using variational principles to characterize coherent vortex structures and giving asymptotic descriptions of the flow. 


\section{Euler Dynamics}

Under the assumption that the fluid is incompressible and ideal, the evolution equations in two-dimensions are given in vorticity form by:

$$
\frac{D \omega}{D t}=\frac{\partial \omega}{\partial t}+\partial(\psi, \omega)=0
$$

with $\psi$ the streamfunction, $\omega$ the (scalar) vorticity and $\partial(\cdot, \cdot)$ the Jacobian derivative.

The circulation round any closed material contour, $\Gamma=\oint \underline{u} \cdot d \underline{l}=\int \omega d A$, is invariant, which is known as Kelvin's circulation theorem (Kelvin, 1869). Applying this to the evolution, it is observed that the fields $\underline{u}(x, t)$ and $\underline{u}(x, 0)$ are 'equivorticity fields'. It has been shown by Arnold [1] that the steady state flows of the system have an extremal energy value in comparison with all close equivorticity flows.

The dynamical equations admit a Hamiltonian (or Poisson) formulation, and by Noether's theorem, the conservation laws for impulse are linked to spatial symmetries of the mathematical model [2]. Then steady motions realize extremal values of the energy for given values of the momentum integral:

$$
\underset{\omega \in \mathcal{A}}{\operatorname{extr}}\{H(\omega) \mid I(\omega)=m\}
$$

where competitors for the conditional extrema are equivorticity fields of the initial flow. The set of rearrangements $\mathcal{A}$ of an initial flow $\omega_{0}$ is given by functions $\zeta$ satisfying an evolution equation of the form:

$$
\frac{\partial \zeta}{\partial t}+\partial(\phi, \zeta)=0
$$

with the initial condition $\zeta(0)=\omega_{0}$ and where $\phi$ is an arbitrary function (satisfying proper boundary conditions). By transporting the vorticity $\omega_{0}$ by an arbitrary velocity field, all vorticity invariants are preserved and so the evolution remains on the isovortical sheets.

The evolution as given by (1) is confined to surfaces of constant energy, but this is not the case for (3) and this idea has been exploited by considering an advecting velocity field such that for the modified dynamics (3) the energy changes monotonically $[14,3,12]$. The numerical algorithm based on the modified equation then converges to conditional extrema of the energy under variations preserving the vortex topology. The method is very useful for searching stable states in a neighbourhood of an initial state or in checking the (Lyapunov) stability of steady state solutions. Since infinitesimally thin filaments cannot be maintained in the numerical simulations, it is possible that stable states are found that would be topologically inaccessible for divergenceless advection. However, assuming that the flow develops inertial vorticity structures at very small scales and that there the effect of a small viscosity will be a filtering on the small scale oscillations of the 
vorticity, it can be expected that the final asymptotic flow can be assimilated with some local average of the vorticity field. For instance, the merging of two like-sign vortices shows a complicated deformation of the vortices, while asymptotically the remaining isolated vortex doesn't seem to depend on the small scale fluctuations in the vorticity field.

This scale separation between the mean flow and the vorticity fluctuations is the underlying idea of the application of statistical mechanics to the $2 \mathrm{D}$ turbulent flows, explaining the emergence of vortices as statistical equilibria $[9,10,11]$. Organized structures appear as states of maximal entropy, where all constants of motion (energy, momentum and the measure of each vorticity level) are taken into account. The final mean flow is a mixing of the initial field, with all the functions of vorticity globally conserved. In this sense, the equilibria resemble the extremal formulation of Arnold, but in fact the initial vorticity lines are allowed to organize into those of the statistical equilibrium, which means that Kelvin's circulation theorem is not satisfied (at the microscopic level) as closed material curves can break and vortices can merge.

For the Euler dynamics when starting from some initial flow, the practical gain of knowing the steady states is limited in the sense that the system will not evolve towards such a constrained critical point of energy or entropy, as both the energy and the vortex topology are conserved for the evolution. The set of critical points is interesting in itself however, since it is invariant for the dynamics and it consists of exact solutions that are stable with respect to certain small perturbations.

It is recognized that any relative equilibrium of the Poisson system (i.e. any constrained critical point of the energy) corresponds to an exact steady solution of the Euler equations. One can consider special families of steady solutions by making a reduction of the dynamics to level sets of some specific invariant integrals. This leads to an adaptation of the variational approach by Amold, considering variations over the set of all admissible vorticity distributions satisfying some appropriate integral constraints:

$$
\underset{\omega \in L_{2}}{\operatorname{extr}}\left\{H(\omega) \mid I(\omega)=m, C_{i}(\omega)=\gamma_{i}\right\}
$$

The relative equilibria are parametrized by only a few (physically relevant) parameters. The Kirchhoff uniform patches form such a special family of steadily rotating vortices, being relative equilibria with constraints for the circulation and the angular momentum. Other examples are given by distributed isolated monopolar vortices $[8,4]$, and dipolar vortices [13].

The variational principles provide a systematic way of finding these special solutions. By posing an additional constraint to the variational principle an unfolding can be made into larger families of steady solutions. The relevance of the families of relative equilibria is emphasized by the 'asymptotic attractivity' for the total flow, if a small dissipation is added to the system. In specific cases a projection can be made of the complete dynamics onto the manifold of relative equilibria. This will be discussed in the next section. 


\section{Self-Organization and Adiabatic Decay}

The idea of selective dissipation and the relation with the asymptotic behaviour of the system are specified by the so-called self-organization hypothesis (see [7] and references therein). In short, if there exist constants of the motion for the conservative part of the system, say $A$ and $B$, for which it holds that in the in the presence of dissipation the integral $A$ dissipates faster than $B$, then the asymptotic behaviour of the solutions of the dissipative system are related to the relative equilibria of minimizing $A$ on level sets of $B$.

A dynamical self-organization principle is obtained by introducing dynamics in the variational characterization of steady state solutions of the conservative system. By projecting on the manifold of relative equilibria, the evolution is described by a principle of dissipating constraint minimizers:

$$
\min _{\omega \in L_{2}}\{A(\omega) \mid B(\omega)=b(t)\}
$$

where the function $b(t)$, representing the dissipation of the integral, can be derived from the evolution equations. Applying this to the 2D NS-equation, asymptotic results have been obtained $[5,6]$, showing that the asymptotic spatial structure of any solution tends to a critical point of the enstrophy on a level set of the energy. The ideal final state of minimum enstrophy may never be reached, since the turbulent motions are themselves diminishing in the process, but the tendency for the turbulent flows to decay towards a state of minimum enstrophy compatible with given energy, expresses the relevance of the relative equilibria [8].

The dynamical self-organization principle can be generalized by adding some suitable constraints to the variational principle. For the 2D flows, minimizers of the enstrophy, given other relevant constraints besides energy, may describe more accurately the intermediate stages of the dynamics. For instance, by specifying the values of different 'moments of vorticity' $\int \omega^{n}$, and studying the relative decay rates, the asymptotic results could be made more precise in the different scales of vorticity. By choosing suitable (non-differentiable) integral constraints, confined vorticity distributions are found, which can be related to the isolated vortices which have been shown to emerge in long time numerical calculations. In this way the adiabatic decay of rotating monopolar vortices has been studied [4], showing the dilating of the vortex domains during the decay process, while the vortex amplitudes gradually diminish. Rotating Kirchhoff ellipses are shown to symmetrize into circular patches. Striking is the observation that maximum energy vortices (rotating anti-cyclonicly) are unstable in the adiabatic decay process, while the minima are stable for the dissipative evolution.

\section{Discussion}

An advantage of Arnold's steady states and the maximum entropy structures compared to the relative equilibria discussed in the previous section, is found in taking 
into account all the constants of motion. However, a prediction of the final steady state needs a precise measurement of the initial conditions (which is hard to achieve in experiments with an initial turbulent flow), and the explicit calculations can become quite difficult in case of an initial vorticity distribution with a large number of vorticity levels. If due to the presence of viscosity the small vorticity fluctuations are dissipated, the integrals measuring the small wavelengths are no longer conserved and the coarse-grained quantities would suffice to determine the equilibrium [9].

For the relative equilibria only a finite number of parameters are used in the variational characterization, which makes the problem topologically easier and the steady states can be found explicitly in many cases. The finite number of Lagrange multipliers makes it possible to consider an explicit adiabatic description of the flow. The functional relationship between the streamfunction $\psi$ and the vorticity $\omega$ can be obtained directly from the variational principles, and in fact any functional relationship can be found by considering the proper integral constraints. The question remains which integrals are relevant for the asymptotics, besides the energy and the enstrophy.

\section{References}

1. Arnold, V.I.: 1966, J. Mec., 5, 29..

2. Benjamin, T.B.: 1984, IMA J. Appl. Math., 32, 3.

3. Carnevale, G.F. and Vallis, G.K.: 1990, J. Fluid Mech., 213, 549.

4. Fliert, van de, B.W. and Groesen, van, E.: 1992, Nonlinearity, 5, 473.

5. Groesen, van, E.: 1987, Phys. A, 148, 312.

6. Groesen, van, E.: 1989, Contemp. Math., 99, 259.

7. Hasegawa, A.: 1985, Adv. Physics, 34, 1.

8. Leith, C.E.: 1984, Phys. Fluids, 27, 1388.

9. Miller, J.: 1990, Phys. Rev. Letters, 65, 2137.

10. Robert, R.: 1990, C.R. Acad. Sci. Paris, 311, 155.

11. Robert, R. and Sommeria, J.: 1991, J. Fluid Mech., 229, 291.

12. Shepherd, T.G.: 1990, J. Fluid Mech.

13. Turkington, B.: 1983, Comm. Part. Diff. Eq., 8, 999.

14. Vallis, G.K. and Carnevale, G.F. and Young, W.R.: 1989, J. Fluid Mech., 207, 133. 